\title{
Deformation induced structural evolution in bulk metallic glasses
}

\author{
WANG Gang ${ }^{1,2^{*}}$, MATTERN Norbert ${ }^{2}$, BEDNARČÍ Jozef ${ }^{3}$, XIA Lei $^{1}$, ZHAI QiJie $^{1}$, \\ DONG YuanDa ${ }^{1} \&$ ECKERT Jürgen ${ }^{2,4}$
}

\author{
${ }^{1}$ Laboratory for Microstructures, Shanghai University, Shanghai 200444, China; \\ ${ }^{2}$ Leibniz-Institute IFW Dresden, Institute for Complex Materials, Dresden D-01069, Germany; \\ ${ }^{3}$ HASYLAB at DESY, Hamburg D-22603, Germany; \\ ${ }^{4}$ TU Dresden, Institute of Materials Science, Dresden D-01062, Germany
}

Received June 2, 2011; accepted August 10, 2011

\begin{abstract}
The structural behavior of binary $\mathrm{Cu}_{50} \mathrm{Zr}_{50}$ and ternary $\mathrm{Cu}_{50} \mathrm{Zr}_{45} \mathrm{Ti}_{5}$ bulk metallic glasses (BMGs) under applied stress was investigated by means of in-situ high energy X-ray synchrotron diffraction. The components of the strain tensors were determined from the shifts of the maxima of the atomic pair correlation functions (PDF) in real space. The anisotropic atomic reorientation in the first-nearest-neighbor shell versus stress suggests structural rearrangements in short-range order. Within the plastic deformation range the overall strain of the metallic glass is equal to the yield strain. After unloading, the atomic structure returns to the stress-free state, and the short-range order is identical to that of the undeformed state. Plastic deformation, however, leads to localized shear bands whose contribution to the volume averaged diffraction pattern is too weak to be detected. A concordant region evidenced by the anisotropic component is activated to counterbalance the stress change due to the atomic bond reorientation in the first-nearest-neighbor shell. The size of the concordant region is an important factor dominating the yield strength and the plastic strain ability of the BMGs.
\end{abstract}

bulk metallic glasses, high energy X-ray diffraction, deformation, structural evolution

Citation: Wang G, Mattern N, Bednarčí J, et al. Deformation induced structural evolution in bulk metallic glasses. Chinese Sci Bull, 2011, 56: 3952-3959, doi: 10.1007/s11434-011-4784-5

Bulk metallic glasses (BMGs) have attracted substantial interest for their superior mechanical properties [1-3]. However, when a BMG is subjected to a sufficiently large stress, it begins to yield and to some extend plastic flow may occur. During this plastic flow, the glass dissipates enormous amounts of energy without breaking. This tolerance of ductile deformation is a crucial requirement in many applications. In the absence of crystallographic defects, such as dislocations or grain boundaries, the fracture strength of metallic glasses has been reported to be close to the theoretical strength of ideal solids [4]. In the past ten years, many BMG systems such as Fe- [5], Co- [6], Ce- [7], and Ca-based BMGs [8] have been developed and the reported strengths vary widely from 300 to $5000 \mathrm{MPa}$. The

*Corresponding author (email: g.wang@ @ shu.edu.cn) strength of a crystalline material is closely related to the Pierce stress, i.e. the intrinsic frictional stress for dislocation motion [9]. The fracture strength of BMGs is believed to be directly associated with the atomic bond because of the lack of crystallographic defects $[1,3]$. Thus, the strength of BMGs is expected to be closely related to the physical parameters determined by the atomic cohesive energy, such as glass transition temperatures, $T_{\mathrm{g}}$, elastic modulus, and thermal expansion coefficients. Understanding of the material parameters governing the strength of the amorphous structure is, therefore, of fundamental importance to the future design of BMGs with desired mechanical properties.

In the deformation process of BMGs, the yield strength of the material depends on the elastic deformation stage. In particular, the atomic structural evolution during elastic deformation can seriously affect the yield strength of mate- 
rials [1]. However, little attendant structural change resulted from elastic deformation and the transient nature of elastic deformation prohibit the operation of structural analysis on the atomic scale in elastic deformation. Since the diffraction technique can measure the lattice plane spacing altered by elastic strain according to Bragg's law, in-situ deformation observed by high energy X-ray diffraction (XRD) provides an effective method for strain scanning on atomic scale metallic glasses [10]. In this paper, we chose the $\mathrm{Cu}-\mathrm{Zr}$ binary BMG family as model materials to explore the correlation between atomic structural evolution driven by stress and macroscopic mechanical properties. This family of BMG has relative simple chemical constitution, which allows us to determine the bond relations, and to correlate them with mechanical properties. By means of in-situ high energy synchrotron X-ray diffraction under compression we were able to follow the atomic reorientation during the elastic and plastic deformation processes.

\section{Experimental procedures}

Alloy ingots with nominal composition of $\mathrm{Cu}_{50} \mathrm{Zr}_{50}$ and $\mathrm{Cu}_{50} \mathrm{Zr}_{45} \mathrm{Ti}_{5}$ (at.\%) were prepared by arc melting a mixture of pure metal elements in a Ti-gettered argon atmosphere, followed by suction casting into a copper mold to form BMG rod samples, diameter $1 \mathrm{~mm}$ and length $20 \mathrm{~mm}$. Compression test samples, $2 \mathrm{~mm}$ long, were cut from these BMG rods with a diamond saw and both ends of the samples were carefully polished to be parallel. The compression tests were carried out on an Instron 8562 type machine at a strain rate of $5 \times 10^{-4} \mathrm{~s}^{-1}$. The elastic parameters, i.e. elastic modulus, shear modulus, bulk modulus and Poisson's ratio, were measured by the ultrasonic technique, which was performed with an Olympus Panametrics NDT 5900PR ultrasonic testing device. The surface morphology of the samples after deformation was observed using a HITACHI TM-1000 scanning electron microscope (SEM).

The samples were compressed using a straining system (Kammrath and Weiss $\mathrm{GmbH}$ ) with a maximum load of 5 $\mathrm{kN}$ and observed in-situ by the X-ray beam at HASYLAB BW5 beamline (at DESY Hamburg, Germany). The applied load was firstly increased to $1200 \mathrm{~N}$ in steps of $200 \mathrm{~N}$ and then further increased to the fracture load in smaller steps of $100 \mathrm{~N}$ or $50 \mathrm{~N}$. The layout of the experimental setup is well illustrated in the schematics of [11]. The strained samples were exposed for $30 \mathrm{~s}$ to the well collimated incident beam with cross section of $0.5 \mathrm{~mm} \times 0.5 \mathrm{~mm}$. Two dimensional XRD patterns $(2300$ pixel $\times 2300$ pixel, $150 \mathrm{~mm} \times 150 \mathrm{~mm}$ pixel size) were collected using a MAR 345 2D image plate detector carefully mounted orthogonal to the X-ray beam. An X-ray beam with a wave length, $\lambda$, of $0.1132 \AA$ was used to register intensity up to a large value $\left(\sim 16.5 \AA^{-1}\right)$ of the scattering vector, $Q,(Q=4 \pi \sin \theta / \lambda)$. The distance between the $2 \mathrm{D}$ detector and the sample was adjusted to $471.75 \mathrm{~mm}$.
The diffraction pattern from $\mathrm{LaB}_{6}$ was used to calibrate the sample to detector distance and tilting of the image plate detector with respect to the beam axis.

\section{Results}

Compression nominal stress-strain curves for two BMGs are shown in Figure 1. The values of Young's modulus and yield strength of the BMGs are listed in Table 1. Compression tests for every specimen were repeated three times to exclude exceptional cases. Both the $\mathrm{Cu}_{50} \mathrm{Zr}_{50}$ and $\mathrm{Cu}_{50} \mathrm{Zr}_{45} \mathrm{Ti}_{5}$ BMGs exhibit significant plasticity after elastic deformation. Other elastic parameters, such as shear modulus (not given), Poisson's ratio and shear wave speed, were obtained by using ultrasonic measurements and are also listed in Table 1.

The stress-displacement curves for in-situ compression using the loading cell fixed in the synchrotron beamline are shown in Figure 2(a), (d). The dark points in these curves represent the strain states at which diffraction patterns were recorded. It is apparent that the structure of the BMGs subjected to the plastic strain could be measured by X-ray diffraction. For the $\mathrm{Cu}_{50} \mathrm{Zr}_{45} \mathrm{Ti}_{5} \mathrm{BMG}$, after plastic deformation and before fracture, the sample was unloaded to $70 \mathrm{MPa}$ to explore the reversible/irreversible nature of the atomic structure evolution. The diffuse diffraction patterns of the BMGs indicate their glassy nature (see insets in Figure 2(a) and (d)). Driven by compressive stress, the diffraction rings become elliptical, i.e. the maxima positions move further apart in the loading direction and closer together in the

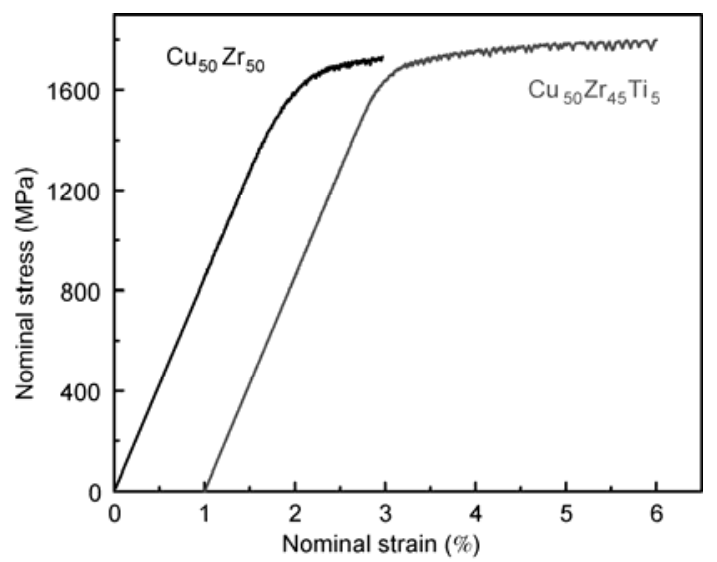

Figure 1 Nominal compressive stress-strain curves for BMGs.

Table 1 Macroscopic mechanical properties of BMGs measured by ultrasonic and compression tests

\begin{tabular}{ccccc}
\hline BMGs & $\begin{array}{c}\text { Elastic modulus } \\
(\mathrm{GPa})\end{array}$ & $\begin{array}{c}\text { Yielding strength } \\
(\mathrm{MPa})\end{array}$ & $\begin{array}{c}\text { Shear wave } \\
\text { speed }(\mathrm{m} / \mathrm{s})\end{array}$ & $\begin{array}{c}\text { Poisson's } \\
\text { ratio }\end{array}$ \\
\hline $\mathrm{Cu}_{50} \mathrm{Zr}_{50}$ & $85 \pm 8$ & $1563 \pm 26$ & $2076 \pm 10$ & 0.378 \\
$\mathrm{Cu}_{50} \mathrm{Zr}_{45} \mathrm{Ti}_{5}$ & $85 \pm 7$ & $1540 \pm 24$ & $2012 \pm 8$ & 0.380 \\
\hline
\end{tabular}



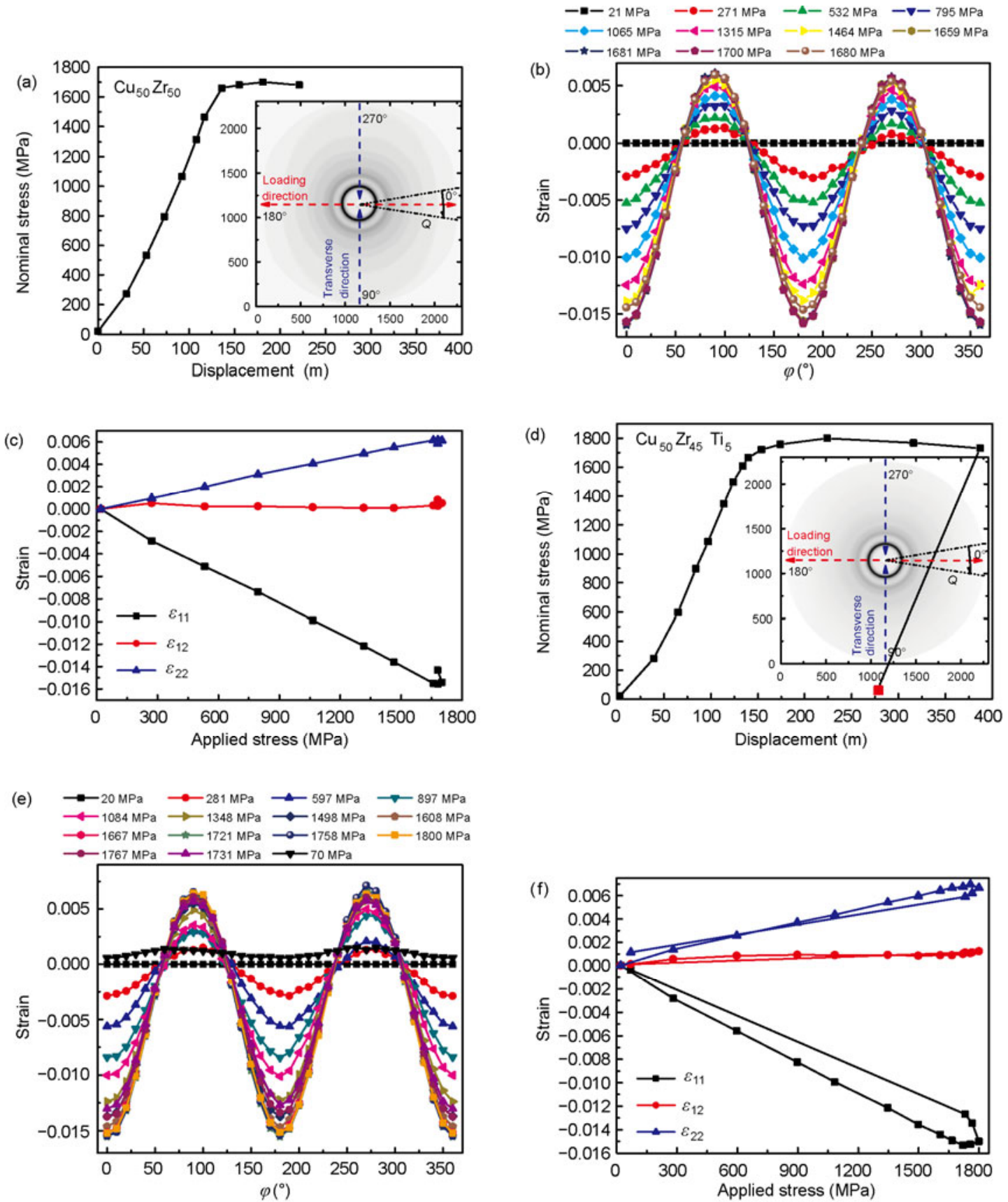

Figure 2 (a) $I n$-situ compression stress-displacement curve and diffraction pattern without stress for $\mathrm{Cu}_{50} \mathrm{Zr}_{50} \mathrm{BMG}$. (b) Direction dependence of the strain values $\varepsilon(\sigma, \varphi)$ for the $\mathrm{Cu}_{50} \mathrm{Zr}_{50}$ BMG. (c) Strain versus stress of the $\mathrm{Cu}_{50} \mathrm{Zr}_{50}$ BMG. Strain values measured from the fit of eq. (1). (d) In-situ compression stress-displacement curve and the diffraction pattern without stress for the $\mathrm{Cu}_{50} \mathrm{Zr}_{45} \mathrm{Ti}_{5}$ BMG. (e) Direction dependence of the strain values $\varepsilon(\sigma, \varphi)$ for the $\mathrm{Cu}_{50} \mathrm{Zr}_{45} \mathrm{Ti}_{5}$ BMG. (f) Strain versus stress of the $\mathrm{Cu}_{50} \mathrm{Zr}_{45} \mathrm{Ti}_{5}$ BMG. Strain values measured from the fit of eq. (1).

transverse direction [12]. The direction dependence of the diffracted intensities is then characterized with respect to polar coordinates, $I(Q, \varphi)$. Using the FIT2D program [13], the diffraction patterns were partitioned into 36 segments. For each $I(Q, \varphi)$, the relative shift of the first peak position, $q_{1}(\sigma, \varphi)$, upon the compressive stress was determined with respect to the unloaded case. The strain values can then be determined by $\varepsilon(\sigma, \varphi)=\left[q_{1}(0, \varphi)-q_{1}(\sigma, \varphi)\right] / q_{1}(\sigma, \varphi)$. The strain values, $\varepsilon(\sigma, \varphi)$ as a function of the direction of $\varphi$ at different stresses are shown in Figure 2(b) and (e). The angular dependence of the curves reflects the symmetry of the uniaxial stress state. From the measured angular variation of the strain the components of the strain tensor, $\varepsilon_{11}, \varepsilon_{22}$ and $\varepsilon_{12}$, were extracted by a least squares fit of $\varepsilon(\sigma, \varphi)=$ $\varepsilon_{11} \sin ^{2} \varphi+\varepsilon_{12} \sin \varphi \cos \varphi+\varepsilon_{22} \cos ^{2} \varphi$. Figure 2(c) and (f) show the calculated values of the loading component, $\varepsilon_{11}$, transverse 
component, $\varepsilon_{22}$, and in-plane shear component, $\varepsilon_{12}$. Linear behavior of the strain with stress is observed up to the yield strength. The shear strain remains almost constant even in the plastic strain regime. The elastic moduli of the $\mathrm{Cu}_{50} \mathrm{Zr}_{50}$ and $\mathrm{Cu}_{50} \mathrm{Zr}_{45} \mathrm{Ti}_{5}$ BMGs measured from the $\varepsilon_{11}$ values are 93 $\pm 2 \mathrm{GPa}$ and $94 \pm 2 \mathrm{GPa}$, respectively (Figure 2(c) and (f)), which are consistent with those measured in the macroscopic compression tests. The Poisson's ratios deduced from the strain values, i.e. $\varepsilon_{22} / \varepsilon_{11}$, are $0.373 \pm 0.006\left(\mathrm{Cu}_{50} \mathrm{Zr}_{50}\right)$ and $0.389 \pm 0.005\left(\mathrm{Cu}_{50} \mathrm{Zr}_{45} \mathrm{Ti}_{5}\right)$, respectively, which are almost the same as those measured using ultrasonic detection
(Table 1).

The intensity curves integrated over sectors at $\varphi=-5^{\circ}-$ $+5^{\circ}$ and $85^{\circ}-95^{\circ}$ were used to represent the structural behaviors in the loading direction and transverse direction, respectively. The intensity curves were then converted into structure factors, $S(Q)$, using standard procedures [14]. The Fourier transform of $S(Q)$ gives the atomic pair correlation function (PDF), $\rho(r)$, i.e. atomic structure information in real space. The PDFs for two BMGs are shown in Figure 3(a). The inset of Figure 3(a) is a detailed view of the first maxima. Besides shifting in the peak positions, doping with
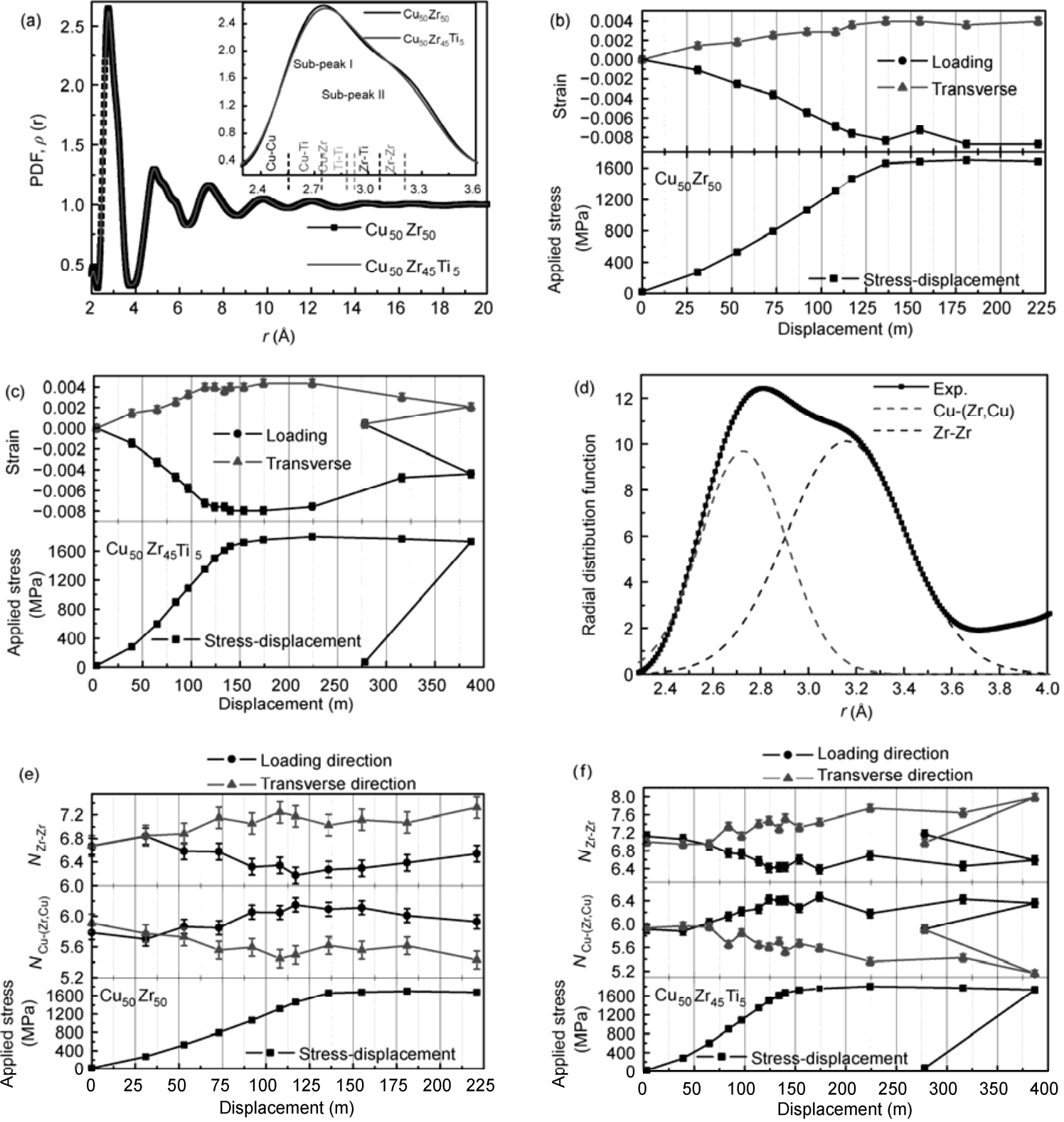

Figure 3 Atomic structural evolution in real space. (a) The PDFs of two $\mathrm{BMGs}$ without stress. Inset shows a comparison of the first maxima from $\mathrm{Cu}_{50} \mathrm{Zr}_{50}$ and $\mathrm{Cu}_{50} \mathrm{Zr}_{45} \mathrm{Ti}_{5}$ BMGs. (b) Deconvolution of the first nearest-neighbor shell into two Gaussians for the Cu $\mathrm{Cu}_{50} \mathrm{Zr}_{50} \mathrm{BMG}$ without stress. (c) Strain values versus displacement of the $\mathrm{Cu}_{50} \mathrm{Zr}_{50}$ BMG measured from the first maximum in the PDF. (d) Strain values versus displacement of the Cu${ }_{50} \mathrm{Zr}_{45} \mathrm{Ti}_{5} \mathrm{BMG}$ measured from the first maximum in the PDF. (e) Coordination numbers versus displacement of the $\mathrm{Cu}_{50} \mathrm{Zr}_{50} \mathrm{BMG}$. (f) Coordination numbers versus displacement of the $\mathrm{Cu}_{50} \mathrm{Zr}_{45} \mathrm{Ti}_{5} \mathrm{BMG}$. 
Ti slightly changes the first-nearest-neighbor shell (see the inset of Figure 3(a)). The first maxima in the PDFs for the two BMGs are composed of two sub-peaks with positions $r_{i}$ at around 2.70 and $3.30 \AA$, respectively (see the inset of Figure 3(a)). For the $\mathrm{Cu}_{50} \mathrm{Zr}_{50} \mathrm{BMG}$, sub-peak I represents overlapping of $\mathrm{Zr}-\mathrm{Cu}$ and $\mathrm{Cu}-\mathrm{Cu}$ pairs, and sub-peak II is assigned to the $\mathrm{Zr}-\mathrm{Zr}$ pair [11]. Doping with Ti brings additional contributions from $\mathrm{Ti}-\mathrm{Ti}, \mathrm{Cu}-\mathrm{Ti}$ and $\mathrm{Zr}$-Ti pairs with theoretical bond lengths of $2.92 \AA, 2.74 \AA$ and $3.06 \AA$, respectively, into the PDF for the $\mathrm{Cu}_{50} \mathrm{Zr}_{45} \mathrm{Ti}_{5}$ BMG. However, the $\mathrm{Cu}-(\mathrm{Zr}, \mathrm{Cu})$ and $\mathrm{Zr}-\mathrm{Zr}$ pairs are the main atomic pairs in both BMGs. The contribution of the other atomic pairs can be neglected because of their lower weighting factors which were calculated for $Q=0 \AA^{-1}$ (Table 2).

Driven by stress, all of the maxima in the PDFs, corresponding to different atomic shells, shift toward smaller $r$ value in the loading direction and toward larger $r$ value in the transverse direction [15]. The strain values measured from the shifts in position of the first maxima are plotted in Figure 3(b) and (c). To compare, the stress-displacement curves are also incorporated into Figure 3(b) and (c). All of the maximum strain limitations of two BMGs in the loading direction are less than -0.010 which is almost half the value measured from the reciprocal space data as shown in Figure 2 , suggesting a small peak shift in the first-nearest-neighbor shell [11]. After yielding, the strain value in the firstnearest-neighbor shell does not significantly change with further increase in macroscopic strain. When the stress is unloaded to around $70 \mathrm{MPa}$ after plastic strain, the strain value in the first-nearest-neighbor shell of the $\mathrm{Cu}_{50} \mathrm{Zr}_{45} \mathrm{Ti}_{5}$ BMG returns to approximately zero (Figure 3(c)).

The integrations over the two sub-peaks of the first maximum of the radial distribution function (RDF), $4 \pi r^{2} \rho(r)$, through Gaussian fitting (Figure 3(d), are related to the atomic nearest-neighbor numbers [11], which are shown in Figure 3(e) and (f). In the loading direction and the elastic deformation regime, the coordination numbers in sub-peak I of both BMGs increase and those in sub-peak II decrease. The opposite behavior happens in the transverse direction. After yielding, the coordination numbers exhibit slight fluctuations in the plastic region. Unloading to $70 \mathrm{MPa}$ stress for the $\mathrm{Cu}_{50} \mathrm{Zr}_{45} \mathrm{Ti}_{5}$ BMG results in the coordination

Table 2 Six possible nearest-neighbor atomic pairs in the two BMGs and their theoretical bond lengths, $R_{i j}^{0}$, and weighting factors at $Q=0 \AA$

\begin{tabular}{cccc}
\hline Atomic pair & $R_{i j}^{0}(\AA)$ & $\begin{array}{c}\text { Weighting factors of } \\
\mathrm{Cu}_{50} \mathrm{Zr}_{50}\end{array}$ & $\begin{array}{c}\text { Weighting factors of } \\
\mathrm{Cu}_{50} \mathrm{Zr}_{45} \mathrm{Ti}_{5}\end{array}$ \\
\hline $\mathrm{Cu}-\mathrm{Cu}$ & 2.70 & 0.177 & 0.186 \\
$\mathrm{Cu}-\mathrm{Zr}$ & 2.90 & 0.487 & 0.462 \\
$\mathrm{Cu}-\mathrm{Ti}$ & 2.75 & & 0.028 \\
$\mathrm{Zr}-\mathrm{Zr}$ & 3.10 & 0.336 & 0.287 \\
$\mathrm{Zr}-\mathrm{Ti}$ & 2.95 & & 0.036 \\
$\mathrm{Ti}-\mathrm{Ti}$ & 2.80 & & 0.001 \\
\hline
\end{tabular}

numbers reversibly changing to the values previously obtained for the state without stress (Figure 3(c)). The total coordination numbers of $\mathrm{Cu}-(\mathrm{Zr}, \mathrm{Cu})$ atomic pairs in the loading direction and the transverse direction for both BMGs, i.e. $\left(N_{\mathrm{Cu}-(\mathrm{Zr}, \mathrm{Cu})}^{\text {loading }}+N_{\mathrm{Cu}-(\mathrm{Zr}, \mathrm{Cu})}^{\text {transverse }}\right) / 2$, remain constant $\left(5.77 \pm 0.07\left(\mathrm{Cu}_{50} \mathrm{Zr}_{50}\right)\right.$ and $\left.5.85 \pm 0.08\left(\mathrm{Cu}_{50} \mathrm{Zr}_{45} \mathrm{Ti}_{5}\right)\right) \mathrm{de}-$ spite applied stress. The numbers of $\mathrm{Zr}-\mathrm{Zr}$ atomic pairs, i.e. $\left(N_{\mathrm{Zr}-\mathrm{Zr}}^{\text {loading }}+N_{\mathrm{Zr}-\mathrm{Zr}}^{\text {transverse }}\right) / 2$, also remain constant at $6.78 \pm$ $0.08\left(\mathrm{Cu}_{50} \mathrm{Zr}_{50}\right)$ and $7.00 \pm 0.09\left(\mathrm{Cu}_{50} \mathrm{Zr}_{45} \mathrm{Ti}_{5}\right)$.

Since the stress can result in an anisotropic structure [11], the PDF also becomes directionally dependent, which can be expressed by expansion into spherical harmonics $[11,12]$ for the case of uniaxial symmetry this expansion is the Legendre polynominal expansion. As such, the PDF is composed of an isotropic component $\left[\rho_{0}(Q)\right]$ and an anisotropic component $\left[\rho_{n}(r)\right]$, which are expressed as [11]:

$$
\begin{gathered}
\rho_{0}(r)=\frac{1}{2 \pi^{2}} \int S_{0}(Q) \frac{\sin (Q r)}{r} Q \mathrm{~d} Q, \\
\rho_{2}(r)=\frac{1}{2 \pi^{2}} \sqrt{\frac{45}{16 \pi}} \int S_{2}(Q) J_{2}(Q r) Q^{2} \mathrm{~d} Q,
\end{gathered}
$$

with $J_{2}(Q r)=\left[\frac{3}{(Q r)^{3}}-\frac{1}{Q r}\right] \sin (Q r)-\frac{3}{(Q r)^{2}} \cos (Q r)$.

Figure 4(a) and (b) compares the calculated isotropic and anisotropic components of the PDFs of two BMGs from the stressless state to the maximum stress. These curves suggest that the difference in the curves of $\rho_{0}(r)$ at different stresses exhibits a small change of approximately $1 \%$ at the first maximum, which can be explained by atomic rearrangement in the short-range under stress [15]. The amplitudes of the maxima in $\rho_{2}(r)$ increase gradually with stress, indicating that anisotropy of the structure is apparent up to about $16 \AA$. After yielding, the curves of $\rho_{2}(r)$ in plastic strain are almost identical (Figure 4(a) and (b)). Unloading the stress from the maximum value $(1800 \mathrm{MPa})$ to $70 \mathrm{MPa}$ for the $\mathrm{Cu}_{50} \mathrm{Zr}_{45} \mathrm{Ti}_{5}$ BMG can reduce the amplitude of the maxima $\rho_{2}(r)$, as clearly evidenced in Figure 4(c) which shows enlargements of the first maxima of $\rho_{2}(r)$ at different stresses.

To characterize the structural behavior in the plastic strain regime, we further observe the sample surface of the BMGs. Figure 5(a) shows the fractured $\mathrm{Cu}_{50} \mathrm{Zr}_{50}$ BMG. Some shear bands are present on the sample surface (see inset of Figure 5(a)). Figure 5(b) gives the morphology of the unloaded $\mathrm{Cu}_{50} \mathrm{Zr}_{45} \mathrm{Ti}_{5}$ BMG. The inset of Figure 5(b) also shows shear bands appearing on the surface of this sample.

\section{Discussion}

The structural information from real space of the $\mathrm{Cu}_{50} \mathrm{Zr}_{45} \mathrm{Ti}_{5}$ 

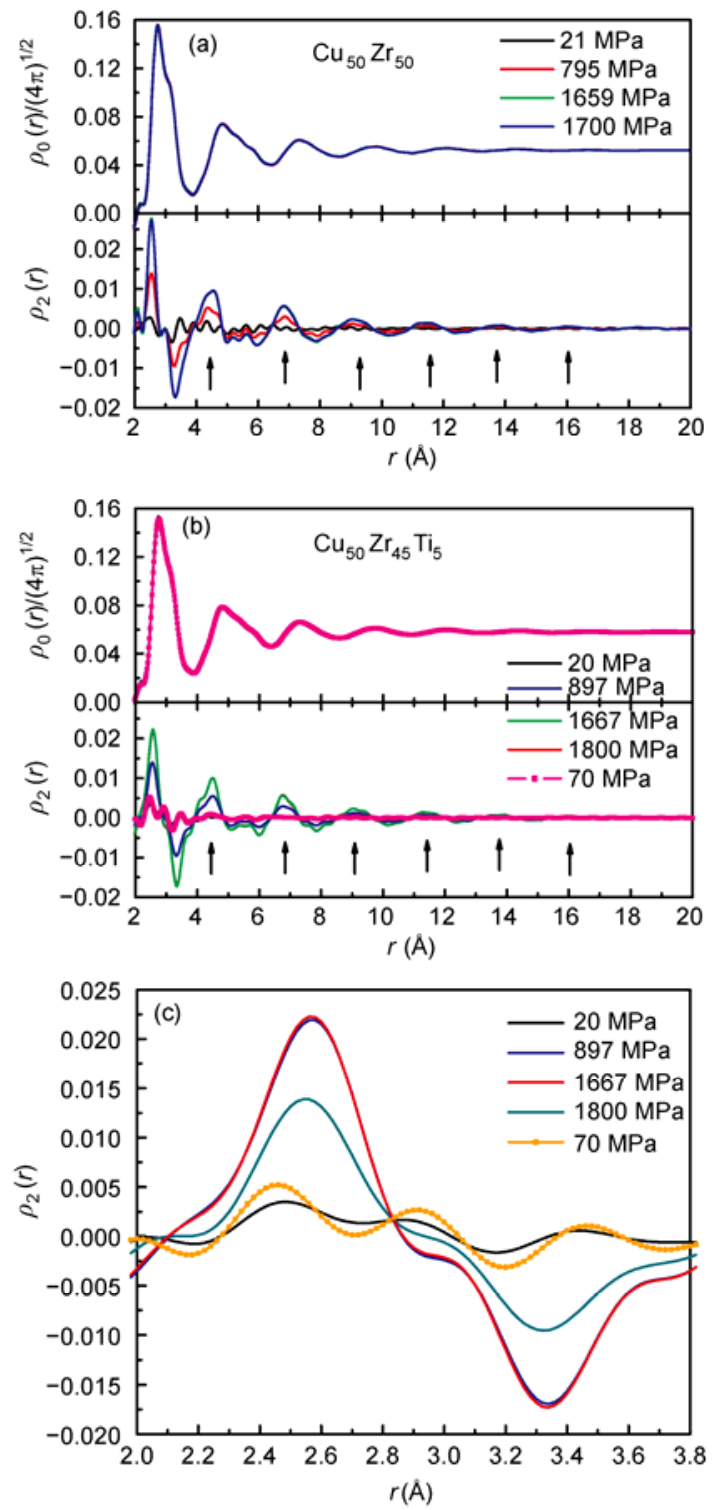

Figure 4 Isotropic component, $\rho_{0}(r)$, and anisotropic component, $\rho_{2}(r)$, of the pair correlation function at different stresses. (a) $\mathrm{Cu}_{50} \mathrm{Zr}_{50}$ BMG; (b) $\mathrm{Cu}_{50} \mathrm{Zr}_{45} \mathrm{Ti}_{5} \mathrm{BMG}$; (c) enlarged anisotropic component, $\rho_{2}(r)$, of the pair correlation function of the $\mathrm{Cu}_{50} \mathrm{Zr}_{45} \mathrm{Ti}_{5} \mathrm{BMG}$ at different stresses.

BMG suggests that even when subjected to a large plastic strain, the atomic anisotropic state observed by the diffraction technique transformed reversibly back to the isotropic state (cf. Figure 4) after unloading. The coordination numbers (cf. Figure 3(f)), and the strain limitations measured from the first-nearest-neighbor shell (cf. Figure 3(c)) and the intensity curves returning to the original state after unloading provide solid evidence. Although the plastic strain behavior introduces a lot of shear bands into the glassy phase, shear strain cannot be observed by our diffraction technique during the plastic strain regime, probably because the volume fraction of the shear bands is too small to give a measurable contribution in the diffraction pattern.

Considering the local atomic environment of the glassy

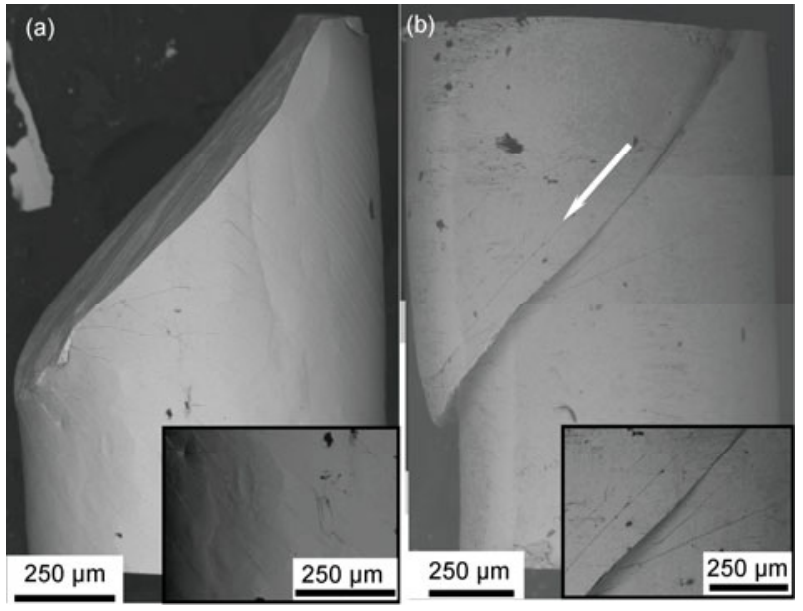

Figure 5 Morphologies of the BMGs subjected to plastic strain. (a) The surface morphology of the $\mathrm{Cu}_{50} \mathrm{Zr}_{50}$ BMG. Inset enlarged to show shear bands. (b) The surface morphology of the $\mathrm{Cu}_{50} \mathrm{Zr}_{45} \mathrm{Ti}_{5}$ BMG. Inset enlarged to show shear bands. The arrows indicate a shear avalanche, which significantly changes the geometry of the BMG.

materials upon loading, a smaller peak shift occurs in the first-nearest-neighbor shell as compared to the peak shifts in the medium-range (cf. Figures 2 and 3). The anisotropic component of the PDFs (Figure 4) and the coordination number calculations suggest that the structural anisotropy occurs because of elastic loading, which is attributed to atomic rearrangements taking place in some topologically unstable regions [16]. A bond reorientation model was deduced to describe this structural anisotropic evolution in glassy materials $[11,17]$. Our calculations of coordination numbers in the first-nearest-neighbor shell suggest that the number of $\mathrm{Cu}-(\mathrm{Zr}, \mathrm{Cu})$ pairs increases along the loading direction and the number of $\mathrm{Zr}-\mathrm{Zr}$ pairs increases along the transverse direction (cf. Figure 3(e) and (f)). According to this, Figure 6 schematically shows the atomic rearrangement resulting in a directional alteration in the first-nearestneighbor shell upon elastic loading. Calculations in Figure $3(\mathrm{e})$ and (f) suggest that the coordination numbers in the
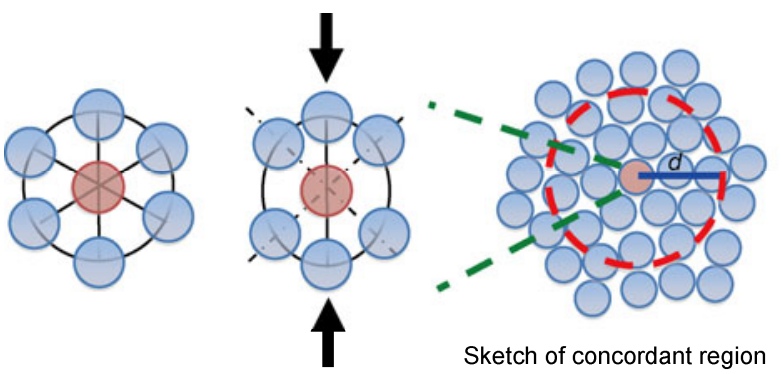

Sketch of atomic reorientation in the first-nearest-neighbor shell upon compression stress

Figure 6 Schematic representations of local bond reorientation resulting in a change in the coordination numbers of the first-nearest-neighbor shell and the atoms concordantly shifting because of the bond reorientation in the first-nearest-neighbor shell. 
plastic strain regime do not remain constant. Two reasons result in the fluctuation of the coordination numbers. One is the force fluctuation due to the serrated flow during plastic strain (cf. Figure 1). Another is the influence of geometrical changes of the BMGs, resulting from the shear band avalanche, as shown in Figure 5, on the diffraction patterns. This geometry change can also shift the diffraction center of the samples, leading to fluctuation of the coordination numbers in the plastic strain regime.

The bond reorientation in the first-nearest-neighbor shell is actually a localized relaxation process reducing the local stress and energy to satisfy the externally applied stress [18]. To counterbalance this reduction in the local stress and energy, other atoms surrounding the first-nearest-neighbor shell will support more stress. As such, this stress increment applied on the surrounding atoms will drive these atoms, within radius $d$, to shift concordantly as shown in Figure 6 [19]. This effect can be considered as a region of concordant relaxation driven by the external stress. This relaxation region can store the elastic energy until yielding. Therefore, the number of atoms within radius $d$ is an important factor dominating the macroscopic mechanical properties.

The stress increment, $\Delta p$, resulting from the bond reorientation, can be expressed as [20]:

$$
\Delta p=\frac{\pi}{2} \Delta p_{0} d_{0}{ }^{3} \rho \ln \frac{2 d}{d_{0}},
$$

where $\rho$ is the mass density, $d_{0}$ is on the order of the size of the first neighbor shell and $\Delta p_{0}$ is the stress increment at the position $d_{0} / 2$. This stress increment significantly increases the activation energy of the concordant region, i.e. $e=e_{0}+$ $V \Delta p$, where $e$ is the activation energy of concordant region, $e_{0}$ is the high-temperature activation barrier and $V$ is the volume of concordant region [21]. The $d$ value in Figure 6 can be calculated by $d=V_{\mathrm{s}} \tau$, where $V_{\mathrm{s}}$ is the shear wave speed and $\tau$ is the relaxation time which depends on the temperature [21]. In the present study, the $\tau$ value is a constant (approximately $10^{-12} \mathrm{~s}$ [19]) since the experiments were carried out at room temperature. The $d$ values for the $\mathrm{Cu}_{50} \mathrm{Zr}_{50}$ and $\mathrm{Cu}_{50} \mathrm{Zr}_{45} \mathrm{Ti}_{5}$ BMGs are calculated to be approximately $20 \AA$ which is consistent with the range $(\sim 16 \AA)$ of the visible anisotropic component in $\rho_{2}(r)$ (cf. Figure 4).

According to the shear wave speeds and the density values listed in Table 1 , the $V$ value of the $\mathrm{Cu}_{50} \mathrm{Zr}_{45} \mathrm{Ti}_{5}$ BMG is $3 \%$ smaller than that of the $\mathrm{Cu}_{50} \mathrm{Zr}_{50}$ BMG. As such, the activation energy of the concordant region, $e$, for $\mathrm{Cu}_{50} \mathrm{Zr}_{45} \mathrm{Ti}_{5}$ is smaller than that for the $\mathrm{Cu}_{50} \mathrm{Zr}_{50}$ BMG. Therefore, a larger elastic energy for the operation of plastic flow in the $\mathrm{Cu}_{50} \mathrm{Zr}_{50}$ BMG is required as compared with that in the $\mathrm{Cu}_{50} \mathrm{Zr}_{45} \mathrm{Ti}_{5} \mathrm{BMG}$, resulting in a higher yield strength for the $\mathrm{Cu}_{50} \mathrm{Zr}_{50} \mathrm{BMG}$. The concordant shifting of the atoms in the concordant region at the yield stress possibly forms a so-called shear transformation zone (STZ). The larger concordant region in the $\mathrm{Cu}_{50} \mathrm{Zr}_{50}$ BMG corresponds to a larger effective STZ compared to that in the $\mathrm{Cu}_{50} \mathrm{Zr}_{45} \mathrm{Ti}_{5}$ BMG [22]. Since the maximum elastic strain required for the formation of a shear band nucleus in BMGs is that which can initiate a local collective motion of STZs [23], a larger STZ volume enables fewer STZs to be activated for the nucleation of a shear band. Therefore, a large concordant region can reduce the shear deformation ability of BMGs, which is consistent with our compression tests (cf. Figure 1). Then, the plastic strain ability of the $\mathrm{Cu}_{50} \mathrm{Zr}_{45} \mathrm{Ti}_{5}$ BMG is higher than that of the $\mathrm{Cu}_{50} \mathrm{Zr}_{50}$ BMG.

\section{Conclusion}

In summary, through in-situ compression tests performed in a high energy $\mathrm{X}$-ray synchrotron radiation beam, the atomic structural evolution of $\mathrm{Cu}_{50} \mathrm{Zr}_{50}$ and $\mathrm{Cu}_{50} \mathrm{Zr}_{45} \mathrm{Ti}_{5}$ BMGs was investigated. The conclusions are:

(1) Under stress, changes in short-range order of the glass occur even during elastic deformation, i.e., the number densities of $\mathrm{Cu}-(\mathrm{Zr}, \mathrm{Cu})$ and $\mathrm{Zr}-\mathrm{Zr}$ nearest-neighbor atomic pairs become oriented relative to the loading direction.

(2) The local deformation in shear bands cannot be seen in the volume averaged PDFs. After unloading, even after plastic deformation, the PDFs are identical within the error limits to those for the as-cast state, which suggests that this anisotropic rearrangement of atoms under stress is a reversible process.

(3) In addition to the atomic bond reorientation in first nearest-neighbor shell, a concordant shift of atoms at medium-range distances occurs with stress, which can dominate the strength of the BMGs. A larger concordant region suggests the operation of a larger STZ, leading to smaller yield strength in the $\mathrm{Cu}_{50} \mathrm{Zr}_{45} \mathrm{Ti}_{5}$ BMG.

G. Wang acknowledges the Alexander von Humboldt Foundation for financial support. The authors thank S. Donath, M. Frey and B. Opitz for technical assistance. This work was supported by the National Natural Science Foundation of China (50731008).

1 Yavari A R, Lewandowski J J, Eckert J. Mechanical properties of bulk metallic glasses. MRS Bull, 2007, 32: 635-638

2 Wang W H. Roles of minor additions in formation and properties of bulk metallic glasses. Prog Mater Sci, 2007, 52: 540-596

3 Chen M W. Mechanical behavior of metallic glasses: Microscopic understanding of strength and ductility. Annu Rev Mater Res, 2008, 38: 445-469

4 Chen H S. Glassy metals. Rep Prog Phys, 1980, 43: 353-432

5 Shen J, Chen Q J, Sun J F, et al. Exceptionally high glass-forming ability of an FeCoCrMoCBY alloy. Appl Phys Lett, 2005, 86: 151907

6 Inoue A, Shen B L, Koshiba H, et al. Cobalt-based bulk glassy alloy with ultrahigh strength and soft magnetic properties. Nat Mater, 2003, 2: 661-663

7 Zhang B, Zhao D Q, Pan M X, et al. Amorphous metallic plastic. Phys Rev Lett, 2005, 94: 205502

8 Senkov O N, Miracle D B, Keppens V, et al. Development and characterization of low-density Ca-based bulk metallic glasses: An overview. Metall Mater Trans A, 2008, 39A: 1888-1900 
9 Gilman J J. Dislocation in Solids. Amsterdam Holland: NorthHolland Press, 1983

10 Hufnagel T C, Ott R T, Almer J. Structural aspects of elastic deformation of a metallic glass. Phys Rev B, 2006, 73: 064204

11 Mattern N, Bednarcik J, Pauly S, et al. Structural evolution of $\mathrm{Cu}-\mathrm{Zr}$ metallic glass under tension. Acta Mater, 2009, 57: 4133-4139

12 Poulsen H F, Wert J A, Neuefeind J, et al. Measuring strain distributions in amorphous materials. Nat Mater, 2005, 4: 33-36

13 Hammersley A P, Svensson S O, Hanfland M, et al. Two-dimensional detector software: From real detector to idealised image or two-theta scan. High Press Res, 1996, 14: 235-248

14 Egami T, Billinge S J L. Underneath the Bragg Peaks: Structural Analysis of Complex Materials. Oxford: Pergamon, New York: Elsevier Press, 2003

15 Wang G, Mattern N, Pauly S, et al. Atomic Structure evolution of bulk metallic glass under compressive stress. Appl Phys Lett, 2009, 95: 251906

16 Srolovitz D, Vitek V, Egami T. An atomistic study of deformation of amorphous metals. Acta Metall, 1983, 31: 335-352

17 Suzuki Y, Haimovich J, Egami T. Bond-orientational anisotropy in metallic glasses observed by X-ray diffraction. Phys Rev B, 1987, 35: 2162-2168

18 Orowan E. Proceedings of the First National Congress of Applied Mechanics. New York: American Society of Mechanical Engineers, 1952

19 Goldstein M. Viscous Liquids and the Glass Transition: A Potential Energy Barrier Picture. J Chem Phys, 1969, 51: 3728-3739

20 Trachenko K. Slow dynamics and stress relaxation in a liquid as an elastic medium. Phys Rev B, 2007, 75: 212201

21 Trachenko K. The Vogel-Fulcher-Tammann law in the elastic theory of glass transition. J Non-crystal Solids, 2008, 354: 3903-3906

22 Pan D, Inoue A, Sakurai T, et al. Experimental characterization of shear transformation zones for plastic flow of bulk metallic glasses. Proc Natl Acad Sci USA, 2008, 105: 14769-14772

23 Johnson W L, Samwer K. A universal criterion for plastic yielding of metallic glasses with a $\left(T / T_{\mathrm{g}}\right)^{2 / 3}$ temperature dependence. Phys Rev Lett, 2005, 95: 195501

Open Access This article is distributed under the terms of the Creative Commons Attribution License which permits any use, distribution, and reproduction in any medium, provided the original author(s) and source are credited. 